\title{
Deficiência da glicose-6-fosfato desidrogenase com infeções de repetição: relato de caso
}

\section{Glucose-6-phosphate dehydrogenase deficiency with recurrent infections: case report}

\author{
Abertina Rosa-Borges ${ }^{1}$, Márcia G. Sampaio ${ }^{2}$, Antônio Condino-Neto ${ }^{3}$, \\ Orlando C.O. Barreto ${ }^{4}$, Victor Nudelman ${ }^{5}$, Magda M.S. Carneiro-Sampaio ${ }^{6}$, \\ Susie A. Nogueira ${ }^{7}$, Thalita F. de Abreu ${ }^{8}$, Jussara Rehder9 ${ }^{9}$, Beatriz T. Costa-Carvalho ${ }^{10}$
}

\section{Resumo}

Objetivo: relatar a ocorrência de uma deficiência funcional de neutrófilos rara, com quadro clínico e laboratorial semelhante ao da doença granulomatosa crônica.

Métodos: relato de caso de paciente com deficiência acentuada da glicose-6-fosfato desidrogenase e infecções de repetição. Realizada pesquisa bibliográfica utilizando as bases de dados Medline e Lilacs, abrangendo o período de 1972 a 2000.

Resultados: paciente com nível da glicose-6-fosfato desidrogenase extremamente reduzido e quadro de infeções graves com melhora clínica após uso de cotrimoxazol contínuo. Os leucócitos do paciente apresentam defeito no metabolismo oxidativo, similar ao da doença granulomatosa crônica.

Conclusões: o diagnóstico da deficiência da glicose-6-fosfato desidrogenase em neutrófilos deve ser considerado em qualquer paciente com anemia hemolítica não esferocítica congênita no qual o nível da glicose-6-fosfato desidrogenase esteja anormalmente baixo ou apresente infeções de repetição. É diagnóstico diferencial da doença granulomatosa crônica.

J Pediatr (Rio J) 2001; 77 (4): 331-6:deficiência de glucosefosfato desidrogenase, síndromes de deficiência imunológica, neutrófilos, doença granulomatosa crônica, anemia hemolítica congênita.

1. Pós-graduanda da Disc. de Alergia, Imunologia Clínicae Reumatologia do Depto. de Pediatria da Univ. Federal de São Paulo (UNIFESP/EPM).

2. Mestranda do curso de Doenças Infecciosas e Parasitárias do Depto. de Medicina Preventiva da Fac. de Medicina da Univ. Federal do Rio de Janeiro (UFRJ).

3. Prof. Assistente Doutor do Centro de Investigação em Pediatria e do Depto. de Pediatria. Faculdade de Ciências Médicas da UNICAMP.

4. Prof. Associado da Fac. de Medicina da Univ. de São Paulo (USP).

5. Pesquisador associado da Disciplina de Alergia, Imunologia Clínica e Reumatologia do Depto. de Pediatria da UNIFESP/EPM.

6. Prof ${ }^{\mathrm{a}}$ Titular do Depto. de Imunologia do Instituto de Ciências Biomédicas da USP.

7. Prof ${ }^{\mathrm{A}}$ Adjunta do Depto. de Medicina Preventiva da Fac. Med. da UFRJ.

8. Prof ${ }^{\mathrm{a}}$ Assistente do Depto. de Pediatria da Fac. de Medicina da UFRJ.

9. Bióloga do Centro de Investigação em Pediatria e Depto. de Pediatria. Faculdade de Ciências Médicas da UNICAMP.

10. Professora Adjunta da Disciplina de Alergia, Imunologia Clínica e Reumatologia do Depto. de Pediatria da UNIFESP-EPM.

\begin{abstract}
Objective: to report a case of rare neutrophil functional disorder with clinical and laboratory findings similar to those of chronic granulomatous disease.

Methods: patient with extremely reduced level of glucose-6phosphate dehydrogenase and recurrent infections that improved after continuous use of cotrimoxazole. The patient presented leukocytes with defective respiratory burst, similar to what occurs in chronic granulomatous disease.

Comments: the diagnosis of glucose-6-phosphate dehydrogenase deficiency in neutrophils should be considered in any patient with hemolytic anemia whose level of G6PD is extremely low or in any patient that presents recurrent infections as differential diagnosis of chronic granulomatous disease.
\end{abstract}

J Pediatr (Rio J) 2001; 77 (4): 331-6: glucosephosphate dehydrogenase deficiency, immunodeficiency syndromes, chronic granulomatous disease, neutrophils, congenital hemolytic anemia.

\section{Introdução}

A deficiência da enzima glicose-6-fosfato desidrogenase (G6PD) afeta mais de 200 milhões de pessoas no mundo. Apresenta freqüência relativamente alta em negros americanos $(13 \%)$ e populações do mediterrâneo $(5 \text { a } 40 \%)^{1}$. Possui herança ligada ao $\mathrm{X}$ e tem como principal manifestação a anemia hemolítica. A hemólise pode ser desencadeada por infeções e por algumas drogas com propriedades oxidativas como o ácido acetil salicílico, a vitamina $\mathrm{K}$, o cloranfenicol e antimaláricos ${ }^{2}$. A G6PD presente nos neutrófilos e eritrócitos é codificada pelo mesmo gene ${ }^{3}$, localizado no cromossomo Xq28 e tende a sofrer freqüentes mutações, tendo sido relatadas mais de 200 variantes ${ }^{4}$. 
Níveis muito baixos de G6PD (abaixo de 5\% do normal) nos neutrófilos podem ser encontrados em algumas raras mutações, levando à falha no metabolismo oxidativo e conseqüente redução da atividade microbicida dependente de oxigênio dos fagócitos. Nesses raros casos, os pacientes com deficiência de G6PD tornam-se susceptíveis a infeções de repetição. Menos de 10 casos foram descritos na literatura. O diagnóstico é estabelecido avaliando o nível da atividade da G6PD nos neutrófilos ${ }^{3}$.

Descrevemos o caso de um paciente com deficiência acentuada de G6PD em eritrócitos e infeções de repetição, tendo sido feita hipótese diagnóstica de imunodeficiência e realizada investigação imunológica.

\section{Relato de Caso}

R.V.S.L, de 5 anos, sexo masculino, pardo, com infeções de repetição desde os 6 meses de vida: pneumonias (12), sendo 2 com derrame pleural, septicemia (1), sinusites (4), otites médias (3), estomatite (1), piodermite (1) e diarréias (2). Necessitou ser internado 8 vezes.

Antecedentes neonatais sem intercorrências. Aleitamento materno exclusivo até 4 meses e misto até 4 anos. Diagnóstico de deficiência de G6PD em eritrócitos com 6 meses de vida. Esquema de vacinação de acordo com o recomendado pelo Ministério da Saúde, sem apresentar reações. Nunca freqüentou escola ou creche.

Pais sem história de consangüinidade. Irmão falecido aos 3 anos por quadro séptico, pneumonia, abscesso pulmonar e hepático. Pai e irmã com traço falciforme.

Ao exame físico apresentava-se pálido, amígdalas hipoplásicas, linfonodos presentes e sem alterações, com baqueteamento de dedos, fígado a $5 \mathrm{~cm}$ do RCD e baço a $8 \mathrm{~cm}$ do RCE.

Avaliação diagnóstica: $\mathrm{Na}$ e $\mathrm{Cl}$ no suor sem alteração, pesquisa para refluxo gastroesofágico negativa, cintilografia pulmonar com ausência de perfusão em pulmão esquerdo, broncoscopia com granuloma.

Hemogramas pregressos: anemia, leucocitose, neutrofilia e eosinofilia; hemograma atual: melhora dos parâmetros hematológicos (Tabela1). Níveis de imunoglobulinas (IgG, IgA, IgM) aumentados (Tabela2), Anticorpo (IgG) positivo para sarampo e rubéola e negativo para vírus da imunodeficiência adquirida. Subpopulacões de linfócitos $\mathrm{CD} 3, \mathrm{CD} 4$ e CD8 com valores normais. Reação de Mantoux de $11 \mathrm{~mm}$. A avaliação do sistema complemento pelo CH50 mostrou atividade normal.

Avaliação de fagócitos: Teste do Nitrobluetetrazolium (NBT): $6 \%$ paciente versus $78 \%$ do controle e da Dihydrorhodamina 123 (DHR): índice de estimulação de 5 vezes no paciente versus 87 vezes no controle.
Tabela 1 - Hemogramas $\left(\mathrm{cel} / \mathrm{mm}^{3}\right)$

\begin{tabular}{llllllll}
\hline $\begin{array}{l}\text { Data/ } \\
\text { HMG }\end{array}$ & Leuc & Neut & Eos & Bas & Linf & Mon & $\begin{array}{l}\text { HB(g/dl)/ } \\
\text { Htc\% }\end{array}$ \\
\hline $02 / 95$ & 20300 & 13601 & 2842 & 0 & 2842 & 1015 & $8 / 28$ \\
$08 / 98$ & 18000 & 11502 & 3132 & 0 & 3078 & 198 & $9,9 / 31,6$ \\
$01 / 99^{*}$ & 15100 & 11627 & 755 & 0 & 2114 & 604 & $10 / 29$ \\
$07 / 00^{*}$ & 7100 & 3053 & 710 & 0 & 3124 & 213 & $10,8 / 33$ \\
\hline
\end{tabular}

*após introdução cotrimoxazol

Leuc $=$ leucócitos, Neut $=$ neutrófilos, Eos $=$ eosinófilos, Bas $=$ basófilos, Linf $=$ linfócitos, Mon = monócitos, $\mathrm{HB}=$ hemoglobina, $\mathrm{Htc}=$ hematócrito

Avaliação da atividade NADPH oxidase dos leucócitos: quantificada a cinética de liberação de superóxido nos tempos $0,5,15,25,45$ e 60 minutos, segundo a redução do citocromo c especificamente inibida pela superóxido dismutase, em condições espontâneas ou mediante estimulação com forbol miristato acetato (PMA, $30 \mathrm{nM}$ ), conforme descrito por Condino-Neto e colaboradores (1996).

Os resultados na Tabela 3 mostram claramente que os leucócitos do paciente com deficiência de G6PD não desencadeiam o burst oxidativo, à semelhança de pacientes com doença granulomatosa crônica.

Dosagem da G6PD em eritrócitos de 1,8 ul/gHb/min a 37 (12,1+/-2,09), demonstrando acentuada deficiência da enzima G6PD.

Feita Hipótese Diagnóstica de deficiência de G6PD. Foi então introduzido cotrimoxazol para uso contínuo em dose terapêutica com controle dos quadros infecciosos, melhora da anemia e da leucocitose e redução do baço.

Tabela 2 - Dosagem de imunoglobulinas (mg/dl)

\begin{tabular}{lcccccccc}
\hline Data/IG & IgG & IgA & IgM & IgE & IgG1 & IgG2 & IgG3 & IgG4 \\
\hline $10 / 95$ & 1767 & 194 & 471 & 60 & & & & \\
$05 / 97$ & & & & & 1130 & 645 & 95 & 2 \\
$07 / 99$ & 2490 & 271 & 320 & 102 & & & & \\
\hline
\end{tabular}

\section{Discussão}

A atividade bactericida dos neutrófilos para microorganismos catalase-positivos depende primariamente de intermediários reativos de oxigênio (ânion superóxido, oxigênio e peróxido de hidrogênio), liberados pela ativação da enzima NADPH oxidase, reação conhecida como metabolismo oxidativo dos neutrófilos ${ }^{5}$. O metabolismo oxidativo 
Tabela 3 - Cinética da liberação de superóxido por leucócitos mononucleares (MON) e polimorfonucleares (PMN) do paciente com deficiência de G6PD (PAC) e de controles sadios (CONTR), nos tempos 0, 5, 15, 25, 45 e 60 minutos, espontânea (ESP) ou estimulada com forbol miristato acetato (PMA, $30 \mathrm{nM}$ )

\begin{tabular}{ccccc}
\hline $\begin{array}{c}\text { Tempo } \\
\text { minutos }\end{array}$ & $\begin{array}{c}\text { MON PAC } \\
\text { ESP }\end{array}$ & $\begin{array}{c}\text { MON PAC } \\
\text { PMA }\end{array}$ & $\begin{array}{c}\text { PMN PAC } \\
\text { ESP }\end{array}$ & $\begin{array}{c}\text { PMN PAC } \\
\text { PMA }\end{array}$ \\
\hline $\mathbf{0}$ & 1.3 & 0.1 & 1.2 & 1.4 \\
$\mathbf{5}$ & 1.1 & 0.2 & 1.5 & 0.5 \\
$\mathbf{1 5}$ & 1.1 & 0.1 & 0.9 & 1.5 \\
$\mathbf{2 5}$ & 0.1 & 0.3 & 0.3 & 0.3 \\
$\mathbf{4 5}$ & 0.1 & 0.3 & 2.1 & 2.2 \\
$\mathbf{6 0}$ & 0.4 & 0.3 & 1.7 & 0.8 \\
\hline Tempo & MON CONTR & MON CONTR & PMN CONTR & PMN CONTR \\
minutos & ESP & PMA & ESP & PMA \\
\hline & & & & \\
$\mathbf{0}$ & 0.2 & 4.3 & 1.1 & 1.0 \\
$\mathbf{5}$ & 0.2 & 9.4 & 0.4 & 2.6 \\
$\mathbf{1 5}$ & 0.2 & 11.6 & 0.3 & 7.1 \\
$\mathbf{2 5}$ & 0.1 & 10.3 & 0.5 & 10.2 \\
$\mathbf{4 5}$ & 1.0 & 9.6 & 0.7 & 1.5 \\
$\mathbf{6 0}$ & 0.6 & 8.7 & 1.9 & 7.0 \\
\end{tabular}

Resultados são a média de dois experimentos, expressos como nmol de superóxido liberado por $10^{6}$ leucócitos

(burst oxidativo é é caracterizado por um aumento abrupto no consumo de oxigênio $\left(\mathrm{O}_{2}\right)$ e sua redução parcial a ânion superóxido $\left(\mathrm{O}_{2-}\right)$. O NADPH é doador específico de elétrons para oxigênio de acordo com a reação:

\section{$\mathrm{NADPH}+\mathbf{2 O}_{2}{ }^{\circledR} \mathrm{NADP}++\mathbf{H}++\mathbf{2 O}_{2-}{ }^{6}$}

$\mathrm{O}_{2-}$, ao agir como um oxidante, é reduzido para peróxido de hidrogênio $\left(\mathrm{H}_{2} \mathrm{O}_{2}\right)^{6}$, que é diretamente tóxico para os microorganismos ${ }^{7}$.

A fonte contínua de NADPH para os fagócitos é a via das hexoses monofosfato. A G6PD é a primeira enzima desta via (hexose monophosphate shunt), onde a glicose-6fosfato (Glucose-6-P) é convertida para 6 fosfogluconato (6-PG) ao mesmo tempo que NADP+ é reduzida para NADPH, substrato para metabolismo oxidativo ${ }^{5}$. Quando o nível de G6PD nos neutrófilos é menor que 5\% do normal $^{3}$, o shunt não é ativado, e a atividade bactericida dos fagócitos é bloqueada.

Na maioria dos tipos de deficiência de G6PD, os níveis nos neutrófilos estão entre 20 e $75 \%$ do normal. Níveis maiores que 5\% de G6PD aparentemente são suficientes para reciclar NADP+ em NADPH em uma velocidade que permite ativação do metabolismo oxidativo e proteção adequada contra infeções por microorganismos ${ }^{3}$.
A enzima NADPH oxidase é composta por vários componentes localizados na membrana plasmática dos fagócitos. A unidade enzimática da NADPH oxidase é composta por uma proteína chamada citocromo B 558 (uma cadeia de 91kDa (gp91phox) e uma cadeia de 22kDa (p22phox)), e duas proteínas do citosol de 47 e 67 kda (p47-phox e p-67-

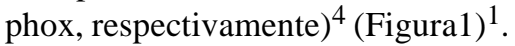

Deficiência de qualquer dos componentes da enzima NADPH oxidase leva ao prejuízo da função microbicida dos fagócitos, em particular contra patógenos catalasepositivos, característica da doença granulomatosa crônica $(\text { DGC })^{8}$.

Portanto, tanto na DGC como na deficiência da G6PD, ocorre um defeito na capacidade bactericida dos neutrófi$\operatorname{los}^{4}$.

Microorganismos produtores de $\mathrm{H}_{2} \mathrm{O}_{2}$ que não contêm catalase (ex. Streptococcus e Haemophilus influenzae) são facilmente destruídos por neutrófilos de pacientes com DGC e deficiência da G6PD ${ }^{6}$, pois seu $\mathrm{H}_{2} \mathrm{O}_{2}$ endógeno não é destruído pela catalase, sendo capaz de suprir as células deficientes com derivados tóxicos de oxigênio.

A atividade do metabolismo oxidativo dos fagócitos pode ser avaliada por métodos bioquímicos e citoquímicos 


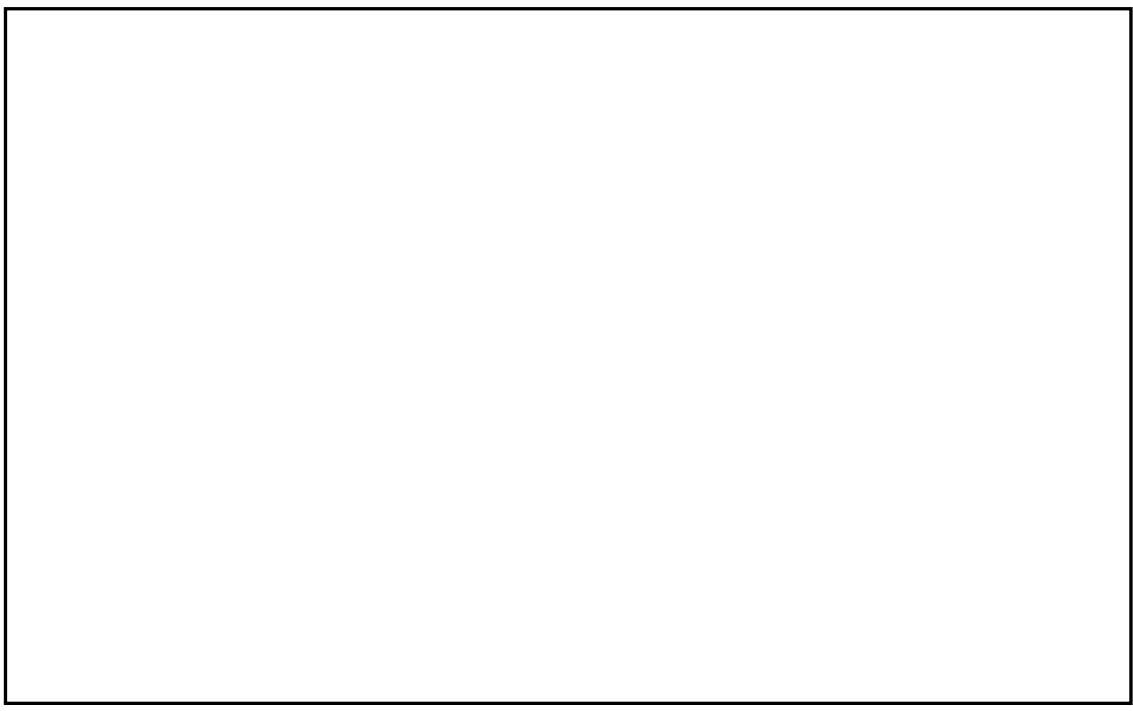

Figura 1 - O complexo NADPH oxidase dos neutrófilos consiste em duas subunidades de membrana do citocromo b, subunidade a $(22 \mathrm{kd})$ e b $(91 \mathrm{kd})$, e dois cofatores citosólicos (67kd e 47kd) necessários para o metabolismo oxidativo. $\mathrm{O}$ NADPH serve como substrato para a redução do $\mathrm{O}_{2}$ em $\mathrm{O}_{2^{-}}$, que é rapidamente transformado em $\mathrm{H}_{2} \mathrm{O}_{2}$ espontaneamente ou pela superóxido dismutase (SOD). O NADPH é gerado a partir da glicose-6-fosfato (glicose6-P) pela glicose-6-fosfato desidrogenase (G6PD) ${ }^{1}$

que detectem a produção de intermediários reativos do oxigênio (ROI). O mais usado é um exame simples e de baixo custo: o teste de redução do corante Nitrobluetetrazo$\operatorname{lium}(\mathrm{NBT})$.

No teste do NBT as células fagocitárias são ativadas por estímulos apropriados na presença do corante (cor amarela), que funciona como receptor de elétrons, e se reduz quando em contato com os intermediários reativos de $\mathrm{O}_{2}$, mudando para a cor azul, podendo ser quantificado no citoplasma dos neutrófilos, facilmente visualizados no microscópio comum ${ }^{6}$.

Outro método para avaliar a atividade do metabolismo oxidativo é a medida direta do consumo de $\mathrm{O}_{2}$, produção de $\mathrm{O}_{2-}$ ou produção $\mathrm{H}_{2} \mathrm{O}_{2}{ }^{9}$. A dosagem específica do $\mathrm{O}_{2-}$ é importante, pois mostra se o defeito no metabolismo oxidativo ocorreu em sua etapa inicial ${ }^{6}$.

A citometria de fluxo também é usada para diagnosticar defeitos do metabolismo oxidativo por apresentar resultados rápidos e precisos ${ }^{10}$. É medida a produção de $\mathrm{H}_{2} \mathrm{O}_{2}$ de neutrófilos ativados com Phorbol-myristate-acetate (PMA), após coloração com Dihydrorhodamina 123 (DHR), substância que se liga aos neutrófilos, causando aumento da fluorescência na presença do $\mathrm{H}_{2} \mathrm{O}_{2}{ }^{11}$.

Embora os métodos de detecção do $\mathrm{O}_{2-}$ e $\mathrm{H}_{2} \mathrm{O}_{2}$ sejam mais sensíveis e específicos para a avaliação do metabolismo oxidativo, o NBT continua sendo o método de referência para screening da $\mathrm{DGC}^{2}$.
Os pacientes com DGC não conseguem reduzir o NBT para a cor azul, e, em algumas variantes raras, é possível detectar fraca redução. Assim como na DGC, quando a deficiência de G6PD é acentuada, o teste do NBT também é alterado ${ }^{4}$ e a produção de $\mathrm{H}_{2} \mathrm{O}_{2}$ e $\mathrm{O}_{2-}$ diminuída.

A DGC é uma deficiência funcional rara, primária, do sistema fagocitário. Geneticamente heterogênea, é caracterizada por infeções graves e de repetição associadas a uma disfunção microbicida dos fagócitos devido a falha no sistema NADPH oxidase ${ }^{8}$. Mutações no gene (localizado no braço curto do cromossomo X) que codifica o flavocitocromo gp 91-phox (componente do sistema NADPH oxidase) é responsável por $60-65 \%$ dos casos de $\mathrm{DGC}^{12}$. O restante apresenta herança autossômica recessiva. A DGC é a disfunção de fagócitos melhor descrita e entendida ${ }^{7}$.

O quadro clínico da deficiência de G6PD, na maioria dos casos, é a anemia hemolítica. Quando a deficiência é acentuada, a apresentação clínica é a mesma da $\mathrm{DGC}^{4}$. Infeções de repetição por microorganismos catalase-positivo (Staphylococcus aureus, enterobactérias como Salmonella, Serratia marcescens, Escherichia coli, Nocardia, fungos como Candida albicans e, principalmente, Aspergi$l l u s^{2}$ ) são freqüentes. Foram relatados casos de septicemia por Chromobacterium violaceum em pacientes com DGC e Deficiência de G6PD, sugerindo que essas disfunções de neutrófilos resultem em uma susceptibilidade à infeção por este patógeno $^{13}$. 
Os locais mais acometidos são pulmões, fígado, pele, trato gastrintestinal e linfonodos. A osteomielite, quando presente, é de resolução difícil. Foram relatadas lesões dos olhos, incluindo córnea e retina. A morte normalmente resulta de infeções fúngicas, principalmente causadas por espécies de Aspergillus ${ }^{9}$.

As bactérias são fagocitadas, mas não são destruídas; dessa forma, esses pacientes tendem a desenvolver abcessos e granulomas crônicos, que podem levar a complicações não infeciosas como hepatoesplenomegalia, linfoadenopatia, hipergamaglobulinemia, diarréia crônica e obstrução granulomatosa em certos órgãos, podendo ser fatais ${ }^{9}$.

Os sintomas e sinais clínicos aparecem durante os dois primeiros anos de vida na maioria dos pacientes. Algumas das crianças apresentam seus primeiros sintomas no período neonatal. Linfoadenopatia está presente em quase todos os casos e, geralmente, é uma das primeiras manifestações da doença. Abcessos hepáticos e periepáticos podem ser, ocasionalmente, os primeiros achados ${ }^{9}$.

O tratamento da deficiência de G6PD é evitar hemólise Em casos acentuados é semelhante ao da DGC, sendo indicada profilaxia com cotrimoxazol, por reduzir a incidência de infeções com risco de vida ${ }^{3,9}$. Apesar do risco de esta droga desencadear hemólise nos pacientes com deficiência de G6PD, por apresentar propriedades oxidativas ${ }^{1}$, estudos e relatos da literatura indicam que o cotrimoxazol raramente causa hemólise na população com deficiência de $\mathrm{G} \mathrm{PD}^{14}$.

A profilaxia com antifúngicos não está indicada, pois apresenta eficácia duvidosa e efeitos colaterais importantes $^{2}$. Deve ser feito uso precoce e agressivo de antibióticos por via parenteral para erradicar infeções ${ }^{3,9}$.

Em infeções com risco de vida, a transfusão de leucócitos pode ser útil, entretanto, tem sido raramente utilizada. A obstrução por granulomas em órgãos vitais pode regredir com o uso de hidrocortisona. Esse tratamento, entretanto, é controverso e deve ser realizado com cautela ${ }^{9}$.

O Interferon-Gama (INF-g) ativa macrófagos in vivo e in vitro, por estimular a NADPH oxidase e aumentar a síntese de intermediários reativos de oxigênio, aumentando sua atividade microbicida em indivíduos saudáveis ${ }^{6}$. A terapia com Interferon Gama recombinante humano (rHuIFN-g) foi recentemente usada em pacientes com DGC com redução do risco relativo de infeções graves em $70 \%$ dos $\operatorname{casos}^{6}$. Embora não tenha reconstituído a atividade metabólica oxidativa na maioria dos pacientes, algumas variantes de DGC - como o defeito na gp91 phox e as deficiências de proteína do citosol - apresentaram melhora na resposta oxidativa ${ }^{8}$. O mecanismo pelo qual $\mathrm{rHuIFN}-\mathrm{g}$ beneficia pacientes com DGC não é ainda totalmente conhecido ${ }^{6,15}$. Sua eficácia na deficiência de G6PD não foi demonstra$\mathrm{da}^{3,9}$.

O paciente relatado apresentou várias infecções bacterianas graves desde os 6 meses de vida. Na maioria delas, necessitou internações prolongadas e antibióticos de largo espectro para controle das infecções, mostrando a necessidade de avaliação do sistema imunológico. Pela gravidade do quadro, entre outros exames, foram realizados os testes do NBT e DHR, para diagnóstico de defeitos funcionais de fagócitos. Ambos se mostraram claramente alterados. Foi então realizada a dosagem da G6PD em função de o paciente apresentar diagnóstico de deficiência de G6PD, realizado aos 6 meses de idade, após investigar quadro de anemia. A dosagem da enzima realizada em eritrócitos demonstrou acentuada deficiência, o que nos fez concluir que os defeitos fagocitários do paciente são causados pela deficiência da G6PD nos neutrófilos. Foi introduzido cotrimoxazol contínuo com melhora clínica importante do paciente, que não apresenta desde então qualquer infecção bacteriana e tampouco sinais clínicos ou laboratoriais de hemólise.

Quando a DGC foi inicialmente diagnosticada, era considerada uma doença granulomatosa fatal. Pacientes hoje diagnosticados têm melhor prognóstico, mas a mortalidade e morbidade ainda são significativas ${ }^{7}$. Apesar do tratamento agressivo, muitos pacientes com DGC morrem antes da adolescência ${ }^{2}$.

O prognóstico da deficiência acentuada de G6PD não é claro pelo pequeno número de casos descritos ${ }^{3}$.

\section{Agradecimentos}

Agradecemos ao Laboratório Fleury pela realização do exame DHR.

\section{Referências bibliográficas}

1. Baehner RL. The Phagocyte System; Chronic Granulomatous Disease. In: Behrman RE, Kliegman RM, Arvin AM, eds. Nelson Textbook of Pediatrics. 15 ${ }^{\text {th }}$ ed. Philadelphia: WB Saunders; 1996.p.586-96.

2. Bellinati-Pires R, Araújo MIAS, Carneiro-Sampaio MMS. Disfunções primárias de neutrófilos: principais aspectos clínicos e laboratoriais. Rev Hosp Clín Fac Med S.Paulo 1992;47:79-85.

3. Curnutte JT. Disorders of granulocyte function and granulopoiesis. In: Nathan DG, Oski FA, eds. Nathan and Oskiфs Hematology of Infancy and Childhood. $4^{\text {th }}$ ed. Philadelphia: WB Saunders;1993. p.904-77.

4. IUIS Scientific Committee: Rosen FS, Eibl M, Roifman C, Fischer A, Volanakis J, Aiuti F, et al. Primary immunodeficiency diseases. Clinical and Experimental Immunology 1999;118:1-28.

5. Ardati KO, Bajakian KM, Tabbara KS. Effect of Glucose-6Phosphate Dehydrogenase on Neutrophil Function. Acta Haematol 1997;97: 211-5.

6. Condino-Neto A, Muscará MN, Bellinati-Pires R, CarneiroSampaio MMS, Brandão AC, Grumach AS, et al. Effect of therapy with recombinant human interferon-g on the release of nitric oxide by neutrophils and mononuclear cells from patients with chronic granulomatous disease. Journal of Interferon and Cytokine Research 1996;16:357-64. 
7. Schiff RI, Harville TO. Primary and secondary immunodeficiency diseases. In: Bierman CW, Pearlman DS, Shapiro GG, Busse WW, eds. Allergy, asthma, and immunology from infancy to adulthood. $3^{\text {rd }}$ ed. Philadelphia: WB Saunders; 1996.p.20-54.

8. Bellinati-Pires R. Avaliação de funções de neutrófilos e monócitos em deficiências primárias e secundárias do sistema fagocitário humano [tese]. São Paulo:USP;1996.

9. Bogomolski-Yahalom V, Matzner Y. Disorders of neutrophil function. Blood reviews 1995;9:183-90.

10. Vowells SJ, Fleisher TA, Sekhsaria S, Alling DW, Maguire TE, Malech H. Genotype-dependent variability in flow cytometric evaluation of reduced nicotinamide adenine dinucleotide phosphate oxidase function in patients with chronic granulomatous disease. The Journal of Pediatrics 1996;128:104-7.

11. Emmendorffer A, Nakamura M, Rothe G, Spiekermann K, Lohmann-Matthes ML, Roesler J. Evaluation of flow cytometric methods for diagnosis of chronic granulomatous disease variants under routine laboratory conditions. Cytometry 1994;18:147-55.

12. Patino PJ, Perez JE, Condino-Neto A, Grumach AS, Botero JH, Curnutte JT, et al. Molecular analysis of chronic granulomatous disease caused by defects in gp91-phox. Hum Mutat 1999;13:29-37.
13. Mamlok RJ, Mamlok V, Mills GC, Daeschner III CW, Schmalstieg FC, Anderson DC. Glucose-6-phosphate dehydrogenase deficiency, neutrophil dysfunction and Chromobacterium violaceum sepsis. The Journal of Pediatrics 1987;111:852-4.

14. Markowitz N, Saravolatz LD. Use of trimethoprim-sulfamethoxazole in a glucose-6-phosphate dehydrogenase-deficient population. Rev Infect Dis 1987;9:S218-29.

15. The International Chronic Granulomatous Disease Cooperative Study Group: Gallin JI, Malech HL, Weening RS, Curnutte JT, Quie PG, Jaffe HS, et al. A controlled trial of interferon gamma to prevent infection in chronic granulomatous disease. The New England Journal of Medicine 1991;324:509-16.

Endereço para correspondência:

Dra. Albertina da Rosa Borges

Rua dos Otonis, 725

CEP 04025-002 - Fone: 5574-0548 / Fax 5579-1590

E-mail: gaai@nox.net 